\title{
Implementation of The Introduction of Skin Diseases Based on Augmented Reality
}

\author{
$1^{\text {st }}$ Mizanuddin Auliya Iqbal \\ Department of Telecommunication \\ Engineering \\ Politeknik Elektronika Negeri Surabaya \\ Surabaya, Indonesia \\ mizandnai@te.student.pens.ac.id
}

\author{
$2^{\text {nd }}$ Akuwan Saleh \\ Department of Telecommunication \\ Engineering \\ Politeknik Elektronika Negeri Surabaya \\ Surabaya, Indonesia \\ akuwan@pens.ac.id
}

\author{
$3^{\text {rd }}$ Haryadi Amran Darwito \\ Department of Telecommunication \\ Engineering \\ Politeknik Elektronika Negeri Surabaya \\ Surabaya, Indonesia \\ amran@pens.ac.id
}

\begin{abstract}
Indonesia is a tropical country that has high rainfall. Environmental conditions greatly affect health conditions in humans such as skin health examples. Many people are still confused to identify the skin disease they are suffering from. This has a negative impact on people's lives. In this research, an application is made that is useful for introducing and informing various types of skin diseases with the Convolutional Neural Network (CNN) algorithm and implementation for information in introducing this skin disease using augmented reality ( $(\mathrm{AR})$. This application is created using the Java programming language and is implemented on smartphones with the Android operating system.

From the test results obtained, the classification results obtained when detecting skin diseases get conditions in accordance with or the same as the trained data. At a light intensity of 355 lux, the highest accuracy was obtained for measles skin disease. For the detection of skin diseases using different distances, the optimal distance to get a high accuracy value is the distance of $8 \mathrm{~cm}$.

Keywords-Convolutional Neural Network, Classification, Augmented Reality, ARCore, Sceneform
\end{abstract}

\section{INTRODUCTION}

The skin is the outermost organ of a human. The skin plays a vital role in protecting organs in the human body from threats originating from the environment outside the human body. Because of this function, the skin becomes sensitive and susceptible to bacterial, viral, and fungal attacks that can cause skin diseases. In Indonesia, the lack of public attention and ignorance of the surrounding environment can cause rapid transmission of skin diseases. So that the speed and accuracy of the diagnosis is very important for treatment, which certainly will affect the patient's recovery and prognosis.

The lack of knowledge about various skin diseases results in frequent misinterpretations of skin diseases suffered by someone. In July 2019, attached from health.detik.com, 11 types of skin diseases that are vulnerable to attack the body where herpes, measles, and acne are included in several types of these diseases. The importance of knowing various skin diseases to anticipate the effects of the skin disease itself. Based on these problems, an idea emerged to create an application to recognize skin diseases through smartphones.

In this research, general concept of this application is the camera will detect the image of existing skin diseases, then analyze and compare them with skin disease data that is already available. After the disease is detected correctly, the camera on the smartphone screen will bring up information and examples of skin diseases suffered on the smartphone screen virtually using Augmented Reality. Augmented reality used in this research is Google's ARCore, where ARcore works to display $3 \mathrm{D}$ models in the form of information in the form of a human body with information about skin diseases around the 3D model of the body. The accuracy of the model is measured by testing the application with several parameters.

\section{SYSTEM OVERVIEW}

This section discusses the methods and materials for implementing systems for the introduction of AR-based skin diseases that are undertaken. Fig. 1 shows the workflow diagram of the proposed classification model. Workflow diagrams are divided into 4 (4); the first discusses data collection, the second phase deals with images and learning of the model, the third phase deals with task classification and performance measurement in applications, and in the fourth section discusses 3D object models displayed using ARCore from Google.

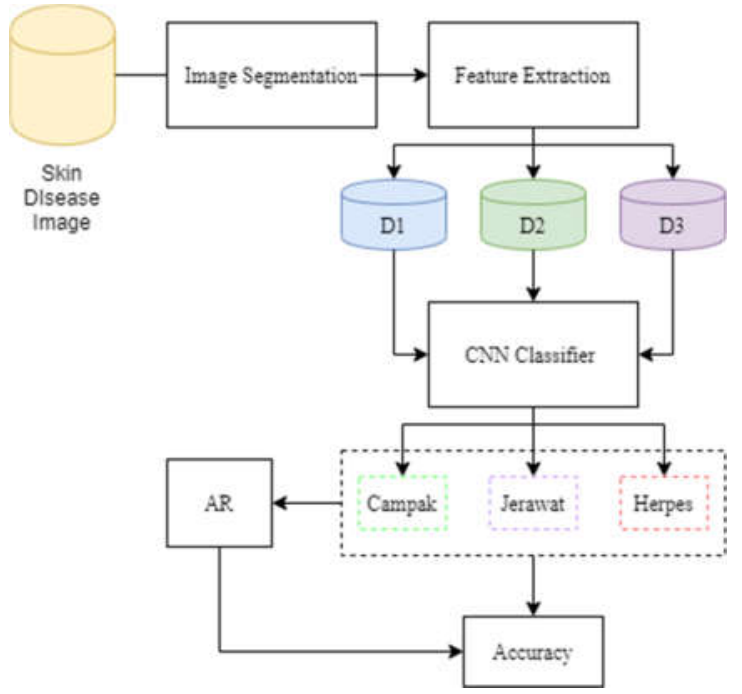

Fig.1. The workflow of the proposed system

\section{A. Dataset and Data preprocessing}

3 skin diseases, namely: measles, acne, and herpes were selected for this study were collected from the internet which is on the health web / which discusses health. Images from the internet have different types of images and the data taken for use as a dataset is taken on images that do not contain watermarks or source writing. 75 images were used in this study with images that met the above criteria. Preprocessing data contributes a lot to the accuracy of the machine learning model. The obtained dataset was preprocessed through data cleaning techniques such as smoothing, fine tuning, aggregation normalization, and attribute construction [4]. 
Table I. Dataset Size

\begin{tabular}{|c|c|}
\hline Skin Diseases & Data Sample \\
\hline Measles & 25 \\
\hline Acne & 25 \\
\hline Herpes & 25 \\
\hline
\end{tabular}

Image Preprocessing : image processing, is the techniques of detection of various images and providing the desired output within type of pictures or the opposite results. Firstly, the aqcuired images were preprocessed and converted to a standar size $(224 \times 224)$ to enhance the image quality and improve the acurracy. Features in the skins such as normal skin without skin diseases are typically regarded as noisy, were filtered off to help the separation of the lesion area from the surrounding skin diseases [3].

Image Segmentation : feature selection is an essential part of machin learning because the performance of the machine learning is dependent on this phase. The feature extraction is done by using the MobileNetV2 architecture which can be used in an android application.

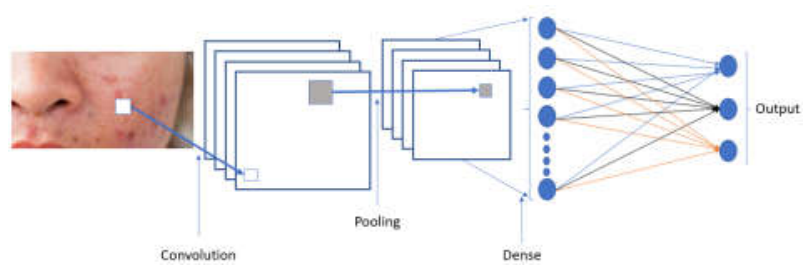

Fig.2. Proposed CNN operation using MobileNetV2

\section{B. Convolutional Neural Network (CNN)}

The Convolutional Neural Network is a deep artificial neural network that is often used to classify images. CNN works by clustering images with similarities and also doing matching schemes with several other schemes. Fig. 2 shows $\mathrm{CNN}$ works by reducing images to a smaller size so that it is easily processed. After the process is carried out, the next scheme is the pooling stage where in this process the average value of each filter is taken from the previous convolution process and continued with the convolution process again to produce a fully connected layer on the output.

The neural network used is MobileNetV2. This architecture contains an initial fully connected layer with 32 filters where this architecture uses ReLU6 as non-linear because of its robustness when used inaccuracy which has little precision in the computation process.

Table II. MobileNetV2

\begin{tabular}{|c|c|c|c|c|c|}
\hline Input & Operator & $t$ & $c$ & $n$ & $s$ \\
\hline $224^{2} \times 3$ & Conv2d & - & 32 & 1 & 2 \\
\hline $112^{2} \times 32$ & bottleneck & 1 & 16 & 1 & 1 \\
\hline $112^{2} \times 16$ & bottleneck & 6 & 24 & 2 & 2 \\
\hline $56^{2} \times 24$ & bottleneck & 6 & 32 & 3 & 2 \\
\hline $28^{2} \times 32$ & bottleneck & 6 & 64 & 4 & 2 \\
\hline $14^{2} \times 64$ & bottleneck & 6 & 96 & 3 & 1 \\
\hline $14^{2} \times 96$ & bottleneck & 6 & 160 & 3 & 2 \\
\hline $7^{2} \times 160$ & bottleneck & 6 & 320 & 1 & 1 \\
\hline $7^{2} \times 320$ & Conv2d 1x1 & - & 1280 & 1 & 1 \\
\hline $7^{2} \times 1280$ & Avgpool 7x7 & - & - & 1 & - \\
\hline $1 \times 1 \times 1280$ & Conv2d $1 \times 1$ & - & - & - & \\
\hline
\end{tabular}

In table 2, each row represents a sequence 1 or more which has multiple identic layers repeated $n$ times. The output channels on each layer are shown at the same number c. All spatial convolutions use a $3 \times 3$ kernel [1].

In MobileNetV2, image input is used with an image resolution and width multiplier that can be tuned so that it can be changed according to the user's wishes for accuracy and performance trade-offs. MobileNetV2 itself prioritizes that the input image used has a width multiplier 1 parameter with a width of 224 pixels and a height of 224 pixels [1].

MobileNetV2 carries out a convolution process for each layer, there are several convolutions, namely depthwise convolution and pointwise convolution. These two convolutions are used for efficiency on the MobileNetV2 architecture where the goal is to change the standard convolution filter into 2 parts. Depthwise convolution convolutes an image with the same height and width as the input, but only 1 dimension is convoluted.

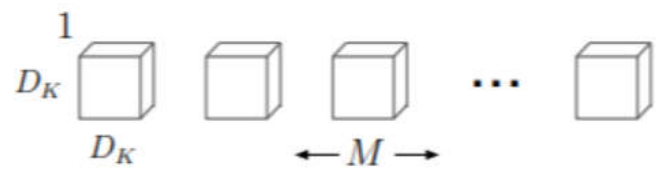

Fig.3. Depthwise convolution filter

After the depthwise convolution filter process, the convolution process is carried out again using a pointwise convolution filter and the convolution process is carried out using the $1 \mathrm{x} 1$ convolutional filter and using the dimensions specified in the MobileNetV2 model architecture.

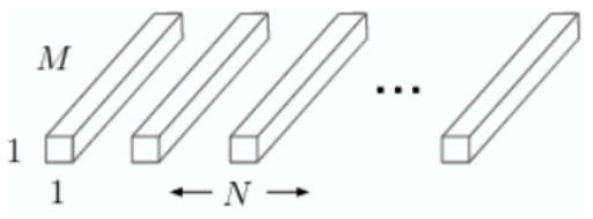

Fig.4. Pointwise convolution filter

To apply the output of the CNN training process, as discussed in the previous discussion that the architecture used is MobileNetV2 [1]. In the process of training the model, the average accuracy of the measurement measure is $80 \%$ in Fig. 5 in a stable position. The results will be imported as a file that is used as a model for classification in Android applications. The model will be used as the main logic for classification to determine when to detect which skin diseases are correct.

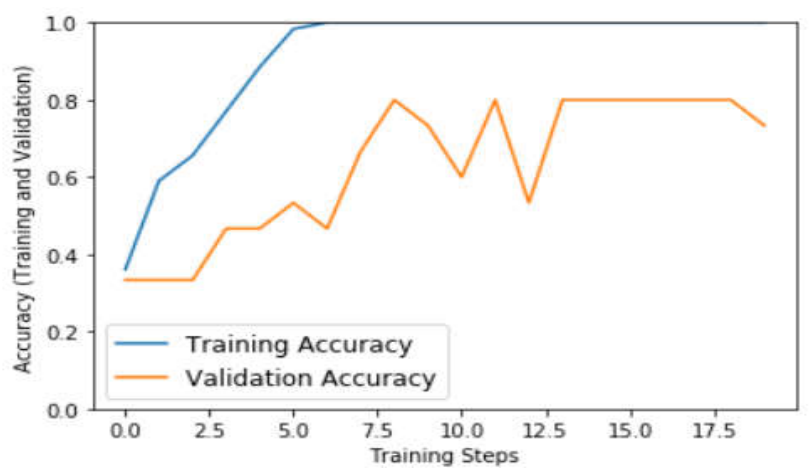

Fig.5. Accuracy of Training 


\section{CNN Model implementation in Android}

The results of the training on CNN have the output in the form of a file. The file is changed to the ".tflite" extension so that it can be used in the android application. This can be done using TensorFlow [2]. The model file which is changed to the ".tflite" extension is used as the main machine learning system in this application.

\section{AR 3D Model Implementation and production}

In the appearance of information on this research using augmented reality technology, where the AR used is ARCore. ARCore can display custom 3D objects that have been created using one of ARCore's tools, the Sceneform. 3D object models are designed using Blender software. In Sceneform, the extension that can be used by Blender software is ".gLTF".

One feature to place 3D objects on Sceneform is to use nodes. Nodes here are used to place custom 3D model objects.

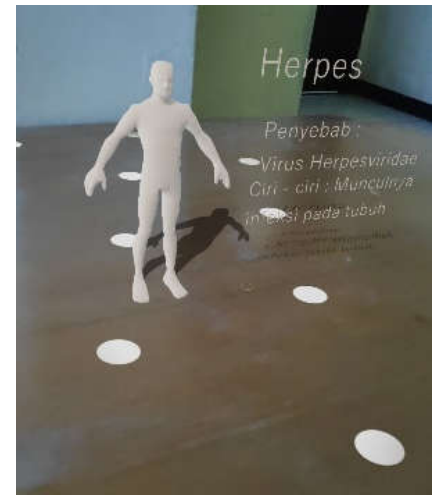

Fig.6. Sceneform for AR Implementation in Android

As in Fig. 6, there are white dots on the media used to place the 3D object where the white dots are nodes. To place the 3D model object manually, click on the area where the 3D model wants to be placed in

\section{RESULT}

There are 3 tests conducted in this study. It is testing for the classification of skin diseases at different light intensities, testing different hearing distances, and testing to display accuracy after the detection process.

\section{A. Testing accuracy at different light intensities}

In the detection of skin diseases, measles skin disease is selected from the three skin diseases detected, and tests are performed on the accuracy results at different light intensities.

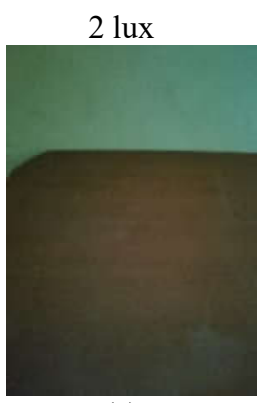

(a)
89 lux

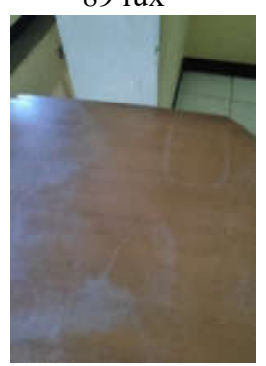

(b)
$248 \operatorname{lux}$

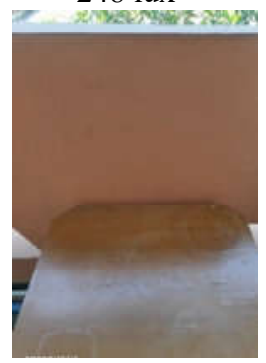

(c)
In Fig. 7, samples were taken at some light intensity in the room. Skin diseases detected in print out and affixed to the wall, then there is a room table that has a function as a medium for placing 3D model objects. Testing is done by detecting images of skin diseases by placing the smartphone at a distance of $8 \mathrm{~cm}$ from images of skin diseases that have been printed out, and aligned on the image so that it is easy to detect images of the skin disease. After the detection is successful, the user can see information for each skin disease with the AR model which is represented in Fig. 4. So the AR model shown in Fig. 4 is used after the user has successfully treated the desired skin disease. This AR model itself will provide information about each skin disease by showing information in the form of writing contained in the AR model.

In Table 3, samples are taken from the test of detection results at 3 different intensities, wherein some of these intensities are light intensity 2 lux, 89 lux, and finally 248 lux. In this test, the distance used to detect measles skin disease is $8 \mathrm{~cm}$ from the image of the skin disease with a smartphone camera. Sampling is done 5 times, where the results of accuracy and detection of the data collection process will be averaged.

From the samples with a light intensity of 2 lux, all the samples obtained had a detection result with an accuracy of $99 \%$, with an average of $99.68 \%$ in 5 tests, all recorded were detected. In testing at 89 Lux light intensity, the detection results at the accuracy obtained are not as good as the samples obtained at 2 Lux light intensity. Accuracy results obtained around $69 \%$ - $83 \%$ with an average accuracy obtained is $96 \%$ detected. In testing at 248 lux light intensity, the detection results with an accuracy of $99 \%$ for 5 times the tests carried out with an average accuracy of $99.91 \%$. Detection results with the most optimal accuracy of the three test models are when taking samples at a light intensity of 248 lux.

TABLE III: Detection of 3 different intensities

\begin{tabular}{|c|c|c|c|c|}
\hline \multicolumn{5}{|c|}{ Skin disease : Measles } \\
\hline \multirow[t]{2}{*}{ Samples } & \multirow[t]{2}{*}{ Range } & \multicolumn{3}{|c|}{ Accuracy } \\
\hline & & $\begin{array}{c}\text { Light } \\
\text { Intensity: } \\
2 \text { lux }\end{array}$ & $\begin{array}{c}\text { Light } \\
\text { Intensity: } \\
89 \text { lux }\end{array}$ & $\begin{array}{c}\text { Light } \\
\text { Intensity: } \\
248 \text { lux }\end{array}$ \\
\hline 1 & $8 \mathrm{~cm}$ & $99,82 \%$ & $69.93 \%$ & $99,98 \%$ \\
\hline 2 & $8 \mathrm{~cm}$ & $99,28 \%$ & $73,48 \%$ & $99,95 \%$ \\
\hline 3 & $8 \mathrm{~cm}$ & $99,73 \%$ & $72,53 \%$ & $99,73 \%$ \\
\hline 4 & $8 \mathrm{~cm}$ & $99,82 \%$ & $80,82 \%$ & $99,96 \%$ \\
\hline 5 & $8 \mathrm{~cm}$ & $99,74 \%$ & $83,29 \%$ & $99,92 \%$ \\
\hline \multicolumn{2}{|c|}{ Average } & $99,68 \%$ & $96,21 \%$ & $99,91 \%$ \\
\hline
\end{tabular}

B. Testing accuracy at different distances

In this test, the detection process is carried out by doing 5 tests at 3 different distances, namely a distance of $8 \mathrm{~cm}, 14 \mathrm{~cm}$, and $20 \mathrm{~cm}$. In this test, the type of skin disease taken as an image for detection is a measles skin disease which is detected in a room with a light intensity of 248 lux.

In Table 4, samples are taken for 3 different distances 5 times. In testing the detection results at a distance of $8 \mathrm{~cm}$, the accuracy obtained is $99 \%$ for 5 times taking samples with an average accuracy of $99.91 \%$. In testing with a distance of $14 \mathrm{~cm}$, the detection results obtained have the accuracy that fluctuates so that there is 1 sample that has a detection result with an accuracy of $79.29 \%$ in the second sample test, and 4 other tests get an accuracy of $91 \%-95 \%$ with an average of -

Fig.7. Testing Place 
the average gets detection results with an accuracy of $91.80 \%$ on testing at a distance of $14 \mathrm{~cm}$. Tests at a distance of $20 \mathrm{~cm}$ were obtained with some incompatible detection results wherein the third and fourth sample testing, there were incorrect detection results with the detection results with an accuracy of $68.44 \%$ detected for herpes but $30.45 \%$ detected for measles skin disease. Whereas in the fourth sample, detection results with an accuracy of $71.51 \%$ were detected for herpes, and $27.31 \%$ were detected for measles skin disease. The other three data get the detection results with an accuracy of $60 \%-86 \%$ with an average accuracy of $56.14 \%$.

Of the three testing models, the average accuracy at a distance of $20 \mathrm{~cm}$ gets the lowest value, where it cannot be used to detect images of skin diseases because the results obtained are not optimal, so to get optimal detection results, detection is carried out at a distance $8 \mathrm{~cm}$ and $14 \mathrm{~cm}$.

TABLE IV: Detection at 3 different distances

\begin{tabular}{|c|c|c|c|}
\hline Samples & $\begin{array}{c}\text { Accuracy } \\
(8 \mathrm{~cm})\end{array}$ & $\begin{array}{c}\text { Accuracy } \\
(14 \mathrm{~cm})\end{array}$ & $\begin{array}{c}\text { Accuracy } \\
(20 \mathrm{~cm})\end{array}$ \\
\hline 1 & $99,98 \%$ & $98,04 \%$ & $85,90 \%$ \\
\hline 2 & $99,95 \%$ & $79,29 \%$ & 76,74 \\
\hline 3 & $99,73 \%$ & $91,09 \%$ & $\begin{array}{c}68,44 \% \text { (Herpes) } / \\
30,45 \% \text { (Measles) }\end{array}$ \\
\hline 4 & $99,96 \%$ & $95,23 \%$ & $\begin{array}{c}71,51 \% \text { (Herpes) } \\
27,31 \% \text { (Measles) }\end{array}$ \\
\hline 5 & $99,92 \%$ & $95,36 \%$ & $60,28 \%$ \\
\hline Average & $99,91 \%$ & $91.80 \%$ & $56,14 \%$ \\
\hline
\end{tabular}

\section{Testing accuracy for different types of diseases}

In this test, 3 types of diseases namely Measles, herpes, and acne will be detected in the room with a light intensity of 248 lux at a distance of $8 \mathrm{~cm}$. Sampling was done 5 times testing.

In Table 5, the detection of measles skin disease results obtained with an accuracy detection with an average of $99.91 \%$ detected. In the detection for acne, the detection results for 5 times the test is unstable. There is a detection result with an accuracy of $88.97 \%$ in the third sample test, while for the other four tests the detection results with an accuracy of $95 \%-99 \%$. The average obtained on the results of acne detection is $95.57 \%$ detected. For testing for herpes, the detection results obtained are $54 \%-94 \%$. The results of this detection are more unstable than the results of the detection of acne. However, from 5 times testing samples, all samples were detected according to skin disease. The average detection result on the accuracy of herpes skin disease was $74.21 \%$.

From the results of tests that have been carried out, all skin diseases at 5 times tested samples have been detected in accordance with their respective skin diseases. These detection results are in accordance with the test results obtained in Table 3 that to get optimal detection results, the distance needed to obtain these results is detection at a distance of $8 \mathrm{~cm}$ and a light intensity of 248 lux.

TABLE V: Detection of 3 types of skin diseases

\begin{tabular}{|c|c|c|c|}
\hline Sample & $\begin{array}{c}\text { Accuracy } \\
\text { (Acne, } 8 \\
\mathrm{~cm})\end{array}$ & $\begin{array}{c}\text { Accuracy } \\
\text { (Measles, } 8 \\
\mathrm{~cm} \text { ) }\end{array}$ & $\begin{array}{c}\text { Accuracy } \\
\text { (Herpes, } 8 \\
\mathrm{~cm} \text { ) }\end{array}$ \\
\hline
\end{tabular}

\begin{tabular}{|c|c|c|c|}
\hline 1 & $95,26 \%$ & $99,98 \%$ & $54,60 \%$ \\
\hline 2 & $97,54 \%$ & $99,95 \%$ & $76,28 \%$ \\
\hline 3 & $88,97 \%$ & $99,73 \%$ & $66,20 \%$ \\
\hline 4 & $98,99 \%$ & $99,96 \%$ & $93,89 \%$ \\
\hline 5 & $97,08 \%$ & $99,92 \%$ & $80,19 \%$ \\
\hline Average & $95,57 \%$ & $99,91 \%$ & $74.21 \%$ \\
\hline
\end{tabular}

D. AR Implementation for Skin Disesases Information

In this section, the 3D object model created will be filled with information about these three skin diseases. In the design for the 3D model itself, it is done using Blender software, which for information displayed, uses the text tool on Blender, and is converted to Mesh so that it can be displayed on the application.

Once converted, the 3D model object is exported into the ".gLTF" extension, and imported into the Android application. To import this extension file, use the tool in ARCore, which is Sceneform, where the file becomes a new extension called ".obj". This extension means that the 3D model object that has been imported has turned into an object that can be detected on Android so that it will produce a new file again with the extension ". sfa" where this file is a file that contains information ranging from name, base color, opacity, sources, and materials used to create the $3 \mathrm{D}$ object model. To use this model, Sceneform will compile the file into a new file again with the extension ".sfb". This file is a binary asset Sceneform that can be used to be built into an android application and loaded at runtime to create the renderable so that when $\mathrm{AR}$ is run, the $3 \mathrm{D}$ model object can be displayed in the real world shown in Fig. 6

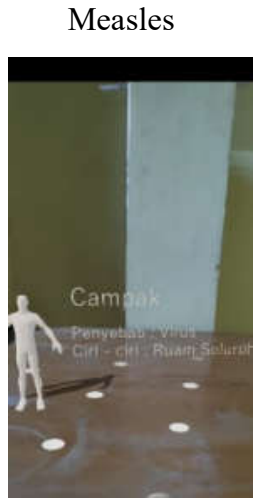

(a)
Herpes

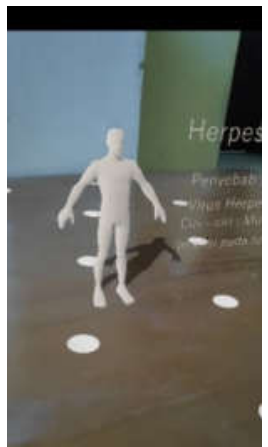

(b)
Acne

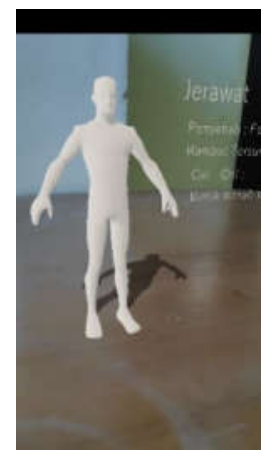

(c)
Fig. 8. 3D Model Object when AR is running

\section{CONCLUSION}

Based on the test results, several conclusions are obtained. Accuracy testing, to get optimal results on detection results, a distance of $8 \mathrm{~cm}$, and a distance of $14 \mathrm{~cm}$ is a distance that can provide detection results with optimal accuracy. Based on the difference in light intensity during testing, namely the light intensity of 2 lux, 89 lux, and 248 lux, in samples taken using measles skin disease, the light intensity of 248 lux gets a detection result with an average accuracy of $99.91 \%$. In the data obtained at a distance of $8 \mathrm{~cm}$, there are also data on some unstable tests so that the detection results with an accuracy of $54.60 \%$, and also $66.20 \%$ as in Table 5 . This can also be affected by the stability of the camera smartphone because even though the application has applied the autofocus feature, 
if the smartphone is slightly unstable, then the focus will be a little blurry, so the detection process gets suboptimal results. To detect the expected optimal results, stability is also needed in the smartphone camera itself so that with a distance of $8 \mathrm{~cm}$ or $14 \mathrm{~cm}$, the light intensity is 248 lux and the smartphone camera is stable, the detection results obtained have high accuracy and have optimal results. if multiple detection is done.

\section{REFERENCES}

[1] Mark Sandler, Andrew Howard, Menglong Zhu, Andrey Zhmoginov, Liang-Chieh Chen, "MobileNetV2: Inverted Residuals and Linear Bottlenecks", Google, 2019.

[2] Mohd Azlan Abu, Nurul Hazirah Indra, Abdul Halim Abd Rahman, Nor Amalia Sapiee and Izanoordina Ahmad, "A study on Image Classification based on Deep Learning and Tensorflow", Universiti Kuala Lumpur British Malaysian Institute, Malaysia, Vol.12, No.4, pp. 563-569, 2019.
[3] Cesar G. Pachón-Suescún, Javier O. Pinzón-Arenas, Robinson Jiménez-Moreno, "Detection of Scratches on Cars by Means of CNN and R-CNN", Department of Mechatronics Engineering, Nueva Granada Military University, Bogotá D.C, 110111, Vol.9, No.3, Colombia.

[4] Nagender Aneja, Sandhya Aneja, "Transfer Learning using CNN for Handwritten Devanagari Character Recognition", Universiti Brunei Darussalam, Brunei Darussalam, 2019. 